Research Paper:

\title{
Prognosis and Survival Study in Patients with Glioblastoma Multiform and Its Relationship With EGFR Expression
}

\author{
Zeinab Amirpour ${ }^{1}$ (D) Arezoo Bahari ${ }^{1}$ (D) Behrad Nafisi $^{1}$ (D) Koorosh Rahmani $^{1}$ (D) Shokouh Taghipour Zahir $^{{ }^{*}}$ (D)
}

1. Shahid Sadoughi General Hospital, Shahid Sadoughi University of Medical Sciences, Yazd, Iran

$\begin{array}{ll}\begin{array}{l}\text { use vour devict to san } \\ \text { and read the article online }\end{array} & \begin{array}{l}\text { dttation: Amirpour Z, Bahari A, Nafisi B, Rahmani K, Taghipour Zahir S. Prognosis and Survival Study in Patients with Glioblas- } \\ \text { toma Multiform and Its Relationship With EGFR Expression. Iran J Neurosurg. 2020; 6(3):113-120. http://dx.doi.org/10.32598/ } \\ \text { irjns.6.3.2 }\end{array} \\ \text { dol: }: \text { http://dx.doi.org/10.32598/irjns.6.3.2 }\end{array}$

\section{(1) (8)}

Article info:

Received: 10 Apr 2020

Accepted: 05 Jun 2020

Available Online: $01 \mathrm{Jul} 2020$

Keywords:

Epidermal growth factor receptor, Glioblastoma multiform, Prognosis

\section{ABSTRACT}

Background and Aim: Glioblastoma Multiforme (GBM) is the most common malignant and invasive tumor of the brain. The relation between prognosis and survival of GBM patients with Epidermal Growth Factor Receptor (EGFR) expression is challenging. Thus, we aimed to evaluate the prognosis and survival of patients with GBM and its relationship with EGFR expression.

Methods and Materials/Patients: This single-arm cohort study was conducted on 70 patients with GBM during 2012-2018 in Shahid Rahnemoon and Mortaz hospitals. The immunohistochemistry technique was applied to paraffin blocks of brain tumors for examining EGFR expression. Other data were extracted from medical records. To determine the survival rate, the Kaplan-Meier curves were used. A chi-square test was used for the analysis of data. Statistically, $\mathrm{P}<0.05$ was assumed significant.

Results: The Mean \pm SD survival of patients with GBM was $22.3 \pm 2.5$ months $(95 \% \mathrm{Cl}=17.41-27.10)$. In addition, 1, 2- and 5-year survival rates were $90 \%, 30 \%$ and $5 \%$, respectively. The mean survival of patients with negative and positive EGFR was $27.4 \pm 7.3$ and $20.6 \pm 2.4$ months, respectively. Besides, $11.1 \%$ and $14.3 \%$ of patients in negative and positive EGFR groups were alive. There was no significant difference in patient's survival in terms of EGFR expression ( $P=0.36)$. No significant difference was seen between the two groups (EGFR positive and negative groups), regarding the frequency of age, sex, tumor's anatomical location, and place of living $(P>0.05)$.

Conclusion: Based on our study, it seems that the GBM tumor was associated with a poor prognosis and a low survival rate. It was also found that the expression of the EGFR gene did not affect the survival rate of patients with GBM. Therefore, its use as a predictor factor for survival and prognosis is questionable.

"Corresponding Author:

Shokouh Taghipour Zahir, MD, AP, CP.

Address: Shahid Sadoughi General Hospital, Shahid Sadoughi University of Medical Sciences, Yazd, Iran

Tel: +98 (913) 3531471

E-mail: taghipourzahirsh@gmail.com 


\section{Highlights}

- Patients with GBM have an overall low survival rate of about 22 months regarding the tumor location.

- No statistical relation was seen between the expression of EGFR (epidermal growth factor receptor) mutation and survival rate.

- No significant relation was seen between the expression of EGFR mutation and patients' age, sex, or the tumor's anatomical location.

\section{Plain Language Summary}

Glioblastoma multiforme is the most malignant brain tumor. Patients with this tumor have little chance of survival, so if neoplastic cells grow more slowly, patients will live 3 to 5 years. Much research has been done on the factors that contribute to the growth of neoplastic cells and the mutations that occur before the aggressive behavior of these cells. Learning about effective molecules and mutations can help us in target therapy and increase survival. One of these mutations seems to be related to the epidermal growth factor receptor in glioblastoma multiforme, which is thought to be mutated in this tumor. If we find a statistical relationship between the expression of this receptor and the survival rate of patients, then we can use the therapeutic goal to slow the growth of malignant cells and thus increase survival. In this study, we examined the expression of this factor and the relationship between its expression and sex, age, tumor's anatomical location, and survival rate. Unfortunately, there was no relationship between the expression of the growth receptor and survival rate, age, sex, and anatomical location. Further studies with more sample sizes are recommended.

\section{Introduction}

lioblastoma Multiforme (GBM) is the most aggressive type of brain tumor with both primary and secondary categories, in which neoplastic cells proliferate rapidly and invade surrounding tissues, disrupting the function of vital centers and resulting in a high mortality rate [1]. The disease usually occurs in the sixth and seventh decades of life. Moreover, the patient's survival rate after the initial diagnosis is $12-15$ months $[2,3]$. A small percentage of patients with GBM can survive 3 years or more $[1,4]$. Despite the improvements in surgical techniques and radiotherapy, the prognosis of these tumors is very poor, thus most patients with GBMs die less than a year and none have long-term survival. Microscopically, GBM shows areas of pseudopalisading necrosis, pleomorphic cells and nuclei, and microvascular proliferation. Genetically, different deletions, point mutations, and amplifications have been observed in GBM, leading to activation of signal transduction pathways downstream of tyrosine kinase receptors such as platelet-derived growth factor receptor and Epidermal Growth Factor Receptor (EGFR) [5]. Studies have shown that EGFR is expressed by approximately $60 \%$ of primary glioblastomas and $10 \%$ of secondary glioblastomas [6]. Mutation of EGFR is observed nearly in one-third of all classical tumors, perineural, and neural mesenchymal glioblastomas [7]. Among these, the mutation of extracellular domain EGFR is observed in glioblastoma [8]. One of the causes of resistance of GMB to therapeutic intervention is the complex feature of the tumor [5]. In the recent years, a new treatment approach has been recognized for the treatment of the disease called target therapy, and those with EGFR mutations appear to respond appropriately to this type of treatment [9]. The study by Smith et al. has found no association between the expression of EGFR with prognosis and survival rate [10]. However, Tripathy et al., have found a significant correlation between the expression of EGFR and survival time [11]. Considering that in previous studies, different results have been reported in terms of EGFR expression and survival rate of patients with GBM, we decided to investigate EGFR expression and its relationship with demographic and prognostic variables and patient survival. If there is a significant relationship between the incidence of this factor and the survival rate of patients, it is suggested to use the anti-EGFR therapy to change the prognosis and survival of patients. 


\section{Methods and Materials/Patients}

This single-arm cohort study was conducted on all patients with GBM during 2012-2018 in Shahid Rahnemoon and Mortaz hospitals, Yazd, Iran. After obtaining written informed consent from patients, the ethical committee of the university approved the study (IR. SSU.MEDICINE.REC.1397.015). Then, brain tumors of patients with GBM who underwent surgery were delivered to the department of pathology. After fixing the tumor in $10 \%$ neutral buffer formalin, they were placed in different concentrations of alcohol (70\%, 80\%, 90\%, and $100 \%)$, xylene, and paraffin solution. Following that, the specimens were sliced into $4 \mu \mathrm{m}$ pieces for staining. The hematoxylin and eosin ( $\mathrm{H} \& \mathrm{E}$ ) were also used to stain and analyze tissue sections. The Immunohistochemistry (IHC) technique was applied on paraffin blocks for staining of EGFR using the EGFR antibody (Dako, Denmark) according to the company protocol. We used a 4-scale scoring system to report the IHC results for expression of EGFR (intensity of staining 0 - no staining, 1 - light, 2 moderate, and 3 - strong) based on the kit protocol and previous studies. All positive staining $(1,2,3)$ were considered positive, and score 0 was considered negative for EGFR expression. Other data including age, sex, survival, and tumor's anatomical location were extracted from medical records. Patients with incomplete medical records were excluded from the study.

\section{Statistical analysis}

Data were entered into SPSS (Version 22, IBM Corp.). The chi-square test was used for data analysis. To deter- mine the survival rate, we used the Kaplan-Meier estimator. The log-rank test was used for comparison of survival. Using PASS 15 software and the median survival in groups and indicators obtained from the log-rank test, the test power was determined to be $80 \%$. Statistically, $\mathrm{P}<0.05$ was assumed significant.

\section{Results}

The aim of this study was to determine the survival of patients with GBM and its association with EGFR. For which, 70 patients were selected, $26(37.1 \%)$ of whom were female and 44(62.9\%) were male. However, data on the survival of 44 patients and EGFR expression of 54 patients were obtained. The survival rate of patients with GBM is shown in Figure 1.

As demonstrated in Figure 1, the mean survival of patients was $22.3 \pm 2.5$ months $(95 \% \mathrm{Cl}=17.41-27.10)$. Most deaths occurred during the first 34 months after diagnosis. Besides, 1, 2- and 5-year survival rate were $90 \%$, $30 \%$ and $5 \%$, respectively. The frequency of patients regarding the tumor's anatomical location, survival rate, place of living, and EGFR expression is shown in Table 1.

As shown in Table 1, 34.9\% of patients had a temporal anatomical location. Moreover, $84.5 .5 \%$ of patients died. The living place of most patients (56.7\%) was Yazd. Also, $41.8 \%$ of patients had a score of +3 for EGFR. The frequency of parameters including age, sex, tumor's anatomical location, and place of living in terms of EGFR expression status is shown in Table 2.

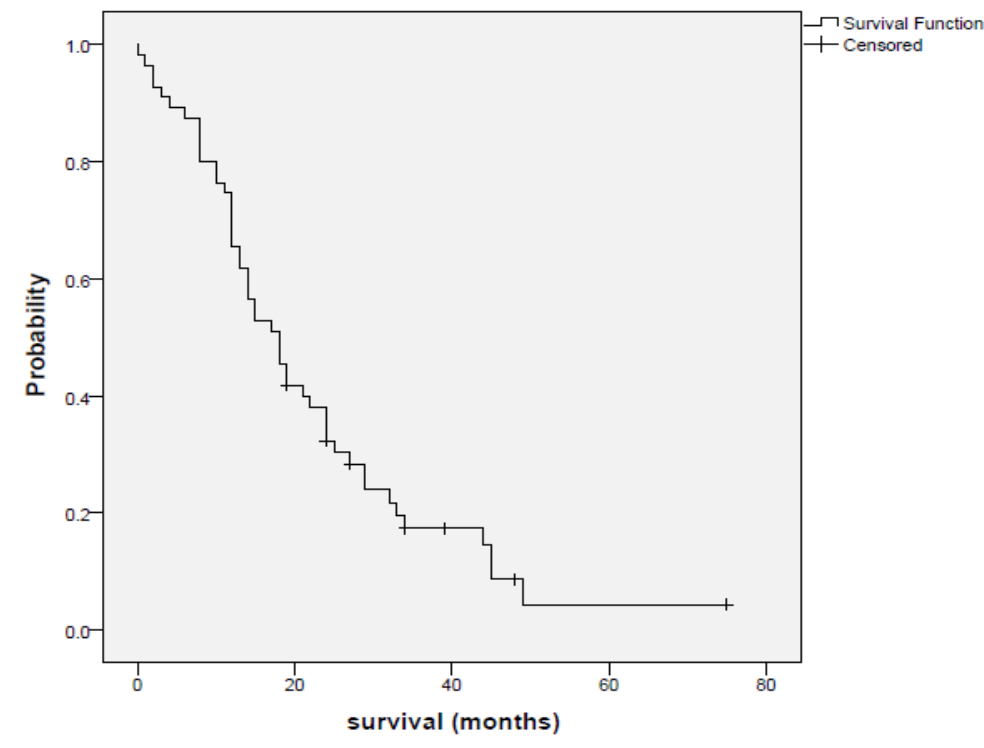

Figure 1. The survival rate of patients with GBM 
Table 1. Frequency of patients, regarding the tumor's anatomical location, survival, place of living, and EGFR expression

\begin{tabular}{|c|c|c|}
\hline \multicolumn{2}{|c|}{ Parameters } & \multirow{2}{*}{$\begin{array}{l}\text { No. (\%) } \\
22(34.9)\end{array}$} \\
\hline \multirow{6}{*}{ Tumor anatomical loc } & Temporal & \\
\hline & Occipital & $11(17.5)$ \\
\hline & Parietal & 12 (19) \\
\hline & Frontal & $12(19)$ \\
\hline & Other & $6(9.5)$ \\
\hline & Total & $63(100)$ \\
\hline \multirow{3}{*}{ Survival } & Dead & 49 (84.5) \\
\hline & Alive & $9(15.5)$ \\
\hline & Total & $58(100)$ \\
\hline \multirow{3}{*}{ Place of living } & Yazd & $38(56.7$ \\
\hline & Other cities & 29 (41.4) \\
\hline & Total & $67(100)$ \\
\hline \multirow{4}{*}{ EGFR expression } & $1+$ & $7(13)$ \\
\hline & $2+$ & $10(18.5)$ \\
\hline & $3+$ & $26(48.1)$ \\
\hline & Negative & 11 (20.4) \\
\hline
\end{tabular}

As shown in Table 2, no significant difference was detected between the two groups regarding the frequency of age, sex, tumor's anatomical location, and place of living ( $P>0.05)$. The mean survival of patients with negative and positive EGFR was $27.4 \pm 7.3$ and $20.6 \pm 2.4$ months, respectively. Furthermore, $11.1 \%$ and $14.3 \%$ of patients in negative and positive EGFR groups were alive. Figure 2 displays the survival rate of patients considering EGFR expression.

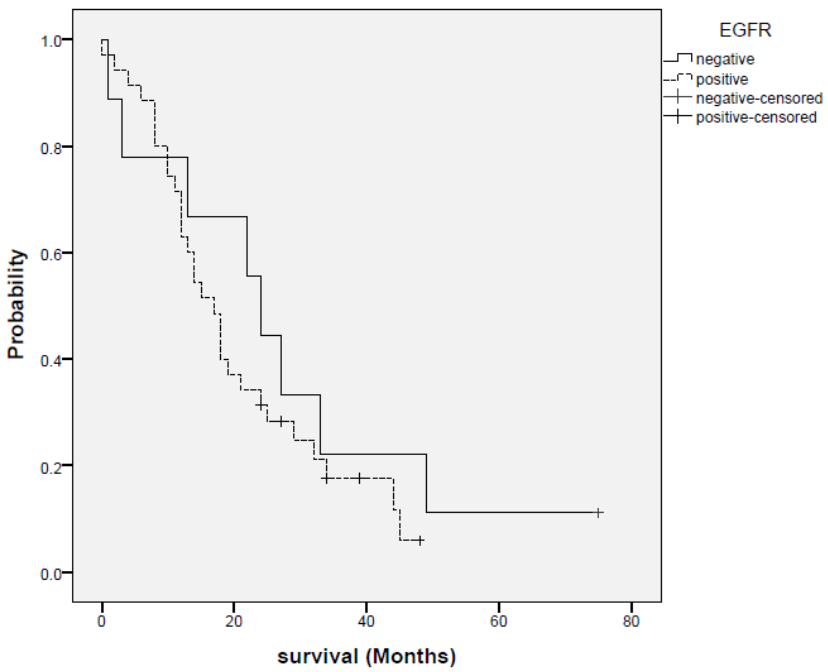

Figure 2. The survival rate of patients considering EGFR expression 
Table 2. Frequency of parameters including age, sex, tumor's anatomical location, and place of living in terms of EGFR expression status

\begin{tabular}{|c|c|c|c|c|}
\hline \multirow{2}{*}{\multicolumn{2}{|c|}{ Parameters }} & \multicolumn{2}{|c|}{ No. (\%) } & \multirow{2}{*}{$\mathbf{P}$} \\
\hline & & EGFR Negative (Group 1) & EGFR Positive (Group 2) & \\
\hline \multirow{3}{*}{ Age (y) } & $20-40$ & $3(30)$ & $7(70)$ & \multirow{3}{*}{0.70} \\
\hline & $40-60$ & $4(18.2)$ & $18(81.8)$ & \\
\hline & $60-80$ & $4(18.2)$ & $18(81.8)$ & \\
\hline \multirow{3}{*}{ Sex } & Female & $4(18.2)$ & $18(81.8)$ & \multirow{3}{*}{0.74} \\
\hline & & & & \\
\hline & Male & $7(21.9)$ & $25(78.1)$ & \\
\hline \multirow{5}{*}{ Tumor's anatomical location } & Temporal & $4(20)$ & $16(80)$ & \multirow{5}{*}{0.45} \\
\hline & Occipital & $2(28.6)$ & $5(71.4)$ & \\
\hline & Parietal & $0(0)$ & $9(100)$ & \\
\hline & Frontal & $1(33.3)$ & $9(66.7)$ & \\
\hline & Other & $1(33.3)$ & $2(66.7)$ & \\
\hline \multirow{3}{*}{ Place of living } & Yazd & 7 (21.9) & $25(78.1)$ & \multirow{3}{*}{0.541} \\
\hline & & & & \\
\hline & Other cities & $3(15)$ & $17(85)$ & \\
\hline
\end{tabular}

As shown in Figure 2, the mean survival of patients in both groups was close. Therefore, there was no significant difference in the survival rate of patients regarding the EGFR expression ( $P=0.36)$. The frequency of parameters including age, sex, tumor's anatomical location, and place of living in terms of EGFR expression is shown in Table 3. As shown in Table 3, no significant difference was observed between all groups (negative, +1 positive, +2 positive, +3 positive), regarding the frequency of age, sex, tumor's anatomical location, and place of living $(P>0.05)$.

\section{Discussion}

GBM is the most malignant type of glial tumor. Despite the low prevalence of this tumor, it accounts for $2.5 \%$ of cancer deaths [12] and the survival of patients is 3 months, but treatment leads to higher survival of these patients. Recently, studies have shown that patients with EGFR mutations have responded appropriately to targeted therapy [11]. In our study, the Mean \pm SD overall survival of patients was $22.3 \pm 2.5$ months. Also, we found that the 1-, 2- and 5-year survival rates of these patients were about $90 \%, 30 \%$, and $5 \%$, respectively. Many studies examined the prognosis and survival of patients with GBM. Krex et al. reported that the mean survival of patients with GBM was 4.6 years [13].

Kumar et al. assessed the survival of patients with GBM and reported that the survival of patients with and without treatment was $12-17$ months and 3 months, respectively [9]. Another study expressed that the mean survival of patients after surgery and resection of the tumor was 12.5 months. In contrast, this time reached 16 months after chemotherapy with temozolomide [13]. Factors including age at diagnosis, low Karnofsky performance status, lack of complete resection of the tumor, and high-grade histological features worsened the prognosis. Surgery and tumor removal had a significant effect on the prognosis and survival of patients [14]. Furthermore, tissue specimens in the current study were assessed considering EGFR expression, and positive EGFR and negative EGFR were observed in 43 (79.6\%) and 11 individuals (20.4\%). No statistically significant difference existed between the duration of survival and the EGFR expression status, which indicates that the expression of EGFR did not affect the survival rate of patients.

In the study by Krex et al., the population of patients with GBM was 55, and EGFR amplification was observed in 10 patients (26\%). The findings unveiled that GBM 
Table 3. Frequency of parameters including age, sex, tumor's anatomical location, and place of living in terms of EGFR expression

\begin{tabular}{|c|c|c|c|c|c|c|}
\hline \multicolumn{2}{|c|}{ Parameters } & Negative & +1 Positive & +2 Positive & +3 Positive & $\mathbf{P}$ \\
\hline \multirow{3}{*}{ Age (y) } & $20-40$ & $3(30)$ & $2(20)$ & $2(20)$ & $3(30)$ & \multirow{3}{*}{0.81} \\
\hline & $40-60$ & $4(18.2)$ & $3(13.6)$ & $5(22.7)$ & $10(45.5)$ & \\
\hline & $60-80$ & $4(18.2)$ & $2(9.1)$ & $3(13.6)$ & $13(59.1)$ & \\
\hline \multirow{2}{*}{ Sex } & Female & $4(18.2)$ & $4(18.2)$ & $2(9.1)$ & $12(54.5)$ & \multirow{2}{*}{0.39} \\
\hline & Male & $7(21.9)$ & $3(9.4)$ & $8(25)$ & $14(43.8)$ & \\
\hline \multirow{5}{*}{$\begin{array}{l}\text { Tumor's anatomical } \\
\text { location }\end{array}$} & Temporal & $4(20)$ & $4(20)$ & $3(15)$ & $9(45)$ & \multirow{5}{*}{0.44} \\
\hline & Occipital & $2(28.6)$ & $0(0)$ & $1(14.3)$ & $4(57.1)$ & \\
\hline & Parietal & $0(0)$ & $1(11.1)$ & $2(22.2)$ & $6(66.7)$ & \\
\hline & Frontal & $1(10)$ & $2(20)$ & $1(10)$ & $6(60)$ & \\
\hline & Other & $1(33.3)$ & $0(0)$ & $2(66.7)$ & $0(0)$ & \\
\hline \multirow{2}{*}{ Place of living } & Yazd & $7(21.9)$ & $3(9.4)$ & $5(15.6)$ & $17(53.1)$ & \multirow{2}{*}{0.505} \\
\hline & Other cities & $3(15)$ & $4(20)$ & $5(25)$ & $8(40)$ & \\
\hline
\end{tabular}

patients with longer survival did not have EGFR amplification. Perhaps because EGFR amplification was more common in elderly patients; however, in this study, patients were young with a long survival [13]. In another study, EGFR amplification was observed in $34 \%$ of patients, and EGFR amplification was associated with a good prognosis in patients with GBM [15].

Rich et al. reported that EGFR expression was not associated with the outcome of patients. It was also found that EGFR amplification was observed in $41 \%$ of patients [16]. Furthermore, EGFRvIll expression was noticed in $55 \%$ of patients. Other studies were conducted in the USA and demonstrated that among 196 patients with GBM, positive EGFR was observed in 105 patients (54\%), negative EGFR in 91 (46\%), and EGFRvIII in 61 (31\%). No significant difference was observed between positive and negative EGFR groups regarding sex [17].

Quan et al. conducted a study on 288 patients with GBM and demonstrated amplification of EGFR in 36 patients (33.6\%). Moreover, no significant relation was seen between the amplification of EGFR and age. It is also believed that the amplification of EGFR was not considered as an influential prognostic factor in patients with GBM [18]. Shinojima et al. proposed that EGFR was expressed in $46 \%$ of patients with GBM. Besides, am- plification of EGFR was reported as a poor prognostic factor in these patients [19].

A study by Smith et al. found no significant differences in survival of patients with or without overexpression of EGFR, which was in line with our study [10]. However, Tripathy et al. have found a significant relationship between overexpression of EGFR and overall survival rate, in which patients without overexpression of EGFR had longer overall survival, being inconsistent with our study [11]. Zhang et al., in their study, concluded that no differences existed between patients with EGFR mutation and patients without overexpression, which was in line with our study [20]. Based on the frequency distribution of patients with GBM, among 70 patients, $15.7 \%$ were in the age range of $20-40$ years, $33(47.1 \%)$ in the age range of $40-60$ years, and $37.1 \%$ in the age range of $60-80$ years. The age range of patients with GBM in various studies was different. Krex et al. reported that the age of patients with GBM ranged from 21 to 72 years old with a mean age of 51 years [13]. Saadeh et al. and Fisher et al. demonstrated that the age range of GBM patients was 18-78 years with a mean age of 54 years old $[21,22]$. Other studies demonstrated the incidence of tumors in the age range of 75-84 years old and mean age 64 years [22]. Still, several researchers stated that age over 50 years was one of the major risk factors for GBM, whereas this disease was rare in the age under 20 
years [23]. GBM occurs in the first 2 decades of life in less than $3 \%$ of all cases [23].

Two different studies were conducted on 174 and 211 patients with GBM and observed only 4 cases with GBM in the first 2 decades of life [24, 25]. In general, according to the mentioned studies, it seems that GBM was more common in the 6 th and 7 th decades of life, and the highest incidence of this tumor was in the age range of 45-65 years, which is consistent with the findings of our study. Also, the frequency of tumors regarding the tumor's anatomical location suggested that the temporal lobe, occipital lobe, parietal lobe, and frontal lobe were observed in $34.9 \%, 17.5 \%, 19 \%$, and $19 \%$ of patients, respectively. Monzón et al. reported that the most common site of tumor involvement was the frontal region. They reported that most symptoms were focal neural symptoms, seizures, and symptoms of increased intracranial pressure [25]. Another study discovered that the most common site of glioblastoma multiforme in the brain was especially in the centers above the tentorium. This tumor makes up $20 \%$ of all intracranial tumors [26].

\section{Conclusion}

Based on the results of the present study, it seems that the GBM tumor was associated with a poor prognosis and a low survival rate. There was no statistically significant relationship between the incidence or absence of EGFR overexpression and survival rate. Besides, there were no significant statistical differences between the status of EGFR expression with age, sex, tumor location, and living place of patients. Therefore, according to these findings, it cannot be used as a predictor factor for patients' survival.

Limitations of the current study were being retrospective, a small sample size, and the lack of evaluation of the effect of monoclonal antibody therapy on patients with overexpression of EGFR.

It is recommended that more comprehensive studies with larger sample sizes as well as clinical trials be carried out on the use of anti-EGFR monoclonal therapy to inhibit this factor and its effect on the patient's prognosis and survival time.

\section{Ethical Considerations}

Compliance with ethical guidelines

This study was approved by the Ethics Committee of the Institutional Ethics Committee of Shahid Beheshti
University of Medical Sciences (Code: IR.SSU.MEDICINE.REC.1397.015). All of the patients gave written informed consent before inclusion in this study.

Funding

This research did not receive any grant from funding agencies in the public, commercial, or non-profit sectors.

\section{Authors' contributions}

Conceptualization and design: Shokouh Taghipour Zahir Data collection: Arezoo Bahari, Koorosh Rahmani; Data analysis and interpretation: Koorosh Rahmani, Shokouh Taghipour Zahir; Drafting the article: Zeinab Amirpour, Arezoo Bahari, Behrad Nafisi, Koorosh Rahmani; Critically revising the article: Shokouh Taghipour Zahir; Reviewing submitted version of manuscript: Zeinab Amirpour, Behrad Nafisi, Koorosh Rahmani, Shokouh Taghipour Zahir; Approving the final version of the manuscript: All authors.

\section{Conflict of interest}

The authors declared no conflict of interest.

\section{Acknowledgments}

The authors would like to thank MS. Pourhosseini for her help in the immunohistochemical staining of the glass slides.

\section{References}

[1] Caruso R, Pesce A, Wierzbicki V. A very rare case report of long-term survival: A patient operated on in 1994 of glioblastoma multiforme and currently in perfect health. International Journal of Surgery Case Reports. 2017; 33:41-3. [DOI:10.1016/j. ijscr.2017.02.025] [PMID] [PMCID]

[2] Lopes MBS, Horten B. Central nervous system tumors. In: Weidner N, Cote RJ, Suster S, Weiss LM, eds. Modern surgical pathology. $1^{\text {st }}$ ed. Philadelphia: WB Saunders; 2003. https://books. google.com/books?id=xmJsAAAAMAAJ\&d

[3] Lefranc F, Brotchi J, Kiss R. Possible future issues in the treatment of glioblastomas: Special emphasis on cell migration and the resistance of migrating glioblastoma cells to apoptosis. Journal of Clinical Oncology. 2005; 23(10):2411-22. [DOI:10.1200/ JCO.2005.03.089] [PMID]

[4] Smoll NR, Schaller K, Gautschi OP. Long-term survival of patients with Glioblastoma Multiforme (GBM). Journal of Clinical Neuroscience. 2013; 20(5):670-5. [DOI:10.1016/j.jocn.2012.05.040] [PMID] 
[5] Holland EC. Glioblastoma multiforme: The terminator. Proceedings of the National Academy of Sciences of the United States of America. 2000; 97(12):6242-4. [DOI:10.1073/pnas.97.12.6242] [PMID] [PMCID]

[6] Xu H, Zong H, Ma Ch, Ming X, Shang M, Li K, et al. Epidermal growth factor receptor in glioblastoma (review). Oncology Letters. 2017; 14(1):512-6. [DOI:10.3892/ol.2017.6221] [PMID] [PMCID]

[7] Verhaak RGW, Hoadley KA, Purdom E, Wang V, Qi Y, Wilkerson $\mathrm{MD}$, et al. Integrated genomic analysis identifies clinically relevant subtypes of glioblastoma characterized by abnormalities in PDGFRA, IDH1, EGFR, and NF1. Cancer Cell. 2010; 17(1):98-110. [DOI:10.1016/j.ccr.2009.12.020] [PMID] [PMCID]

[8] Ohgaki H, Kleihues P. Genetic alterations and signaling pathways in the evolution of gliomas. Cancer Science. 2009; 100(12):2235-41. [DOI:10.1111/j.1349-7006.2009.01308.x] [PMID]

[9] Klatt EC, Kumar V. Robbins and Cotran review of pathology. $9^{\text {th }}$ ed. Elsevier: Sanders Publisher; 2015. https://www. google.com/books/edition/Robbins_and_Cotran_Review_ of_Pathology/jIKICgAAQBAJ?hl=en\&gbpv=0

[10] Smith JS, Jenkins RB. Genetic alterations in adult diffuse glioma: Occurrence, significance, and prognostic implications. Frontiers in Bioscience. 2000; 5:D213-31. [DOI:10.2741/ Smith] [PMID]

[11] Tripathy K, Das B, Singh AK, Misra A, Misra S, Misra SS. Prognostic significance of epidermal growth factor receptor in patients of glioblastoma multiforme. Journal of Clinical and Diagnostic Research. 2017; 11(8):EC05-8. [DOI:10.7860/ JCDR/2017/30138.10327] [PMID] [PMCID]

[12] Hanif F, Muzaffar K, Perveen K, Malhi SM, Simjee SU. Glioblastoma multiforme: A review of its epidemiology and pathogenesis through clinical presentation and treatment. Asian Pacific Journal of Cancer Prevention. 2017; 18(1):3-9. [DOI:10.22034/APJCP.2017.18.1.3] [PMID] [PMCID]

[13] Krex D, Klink B, Hartmann Ch, von Deimling A, Pietsch T, Simon M, et al. Long-term survival with glioblastoma multiforme. Brain. 2007; 130(10):2596-606. [DOI:10.1093/brain/ awm204] [PMID]

[14] Lacroix M, Abi-Said D, Fourney DR, Gokaslan ZL, Shi W, DeMonte F, et al. A multivariate analysis of 416 patients with glioblastoma multiforme: Prognosis, extent of resection, and survival. Journal of Neurosurgery. 2001; 95(2):190-8. [DOI:10.3171/jns.2001.95.2.0190] [PMID]

[15] Houillier C, Lejeune J, Benouaich-Amiel A, Laigle-Donadey F, Criniere E, Mokhtari K, et al. Prognostic impact of molecular markers in a series of 220 primary glioblastomas. Cancer. 2006; 106(10):2218-23. [DOI:10.1002/cncr.21819] [PMID]

[16] Rich JN, Hans Ch, Jones B, Iversen ES, McLendon RE, Ahmed Rasheed BK, et al. Gene expression profiling and genetic markers in glioblastoma survival. Cancer Research. 2005; 65(10):4051-8. [DOI:10.1158/0008-5472.CAN-04-3936] [PMID]

[17] Heimberger AB, Hlatky R, Suki D, Yang D, Weinberg J, Gilbert M, et al. Prognostic effect of epidermal growth factor receptor and EGFRvIII in glioblastoma multiforme patients. Clinical Cancer Research. 2005; 11(4):1462-6. [DOI:10.1158/1078-0432.CCR-04-1737] [PMID]
[18] Quan AL, Barnett GH, Lee SY, Vogelbaum MA, Toms SA, Staugaitis SM, et al. Epidermal growth factor receptor amplification does not have prognostic significance in patients with glioblastoma multiforme. International Journal of Radiation Oncology, Biology, Physics. 2005; 63(3):695-703. [DOI:10.1016/j.ijrobp.2005.03.051] [PMID]

[19] Shinojima N, Tada K, Shiraishi Sh, Kamiryo T, Kochi M, Nakamura H, et al. Prognostic value of epidermal growth factor receptor in patients with glioblastoma multiforme. Cancer Research. 2003; 63(20):6962-70. [PMID]

[20] Zhang LY, Ge HJ, Wang LM, Zhao LH, Liu L, Zhang DJ, et al. [Prognostic implication of alterations in epidermal growth factor receptor and MGMT in glioblastoma (Chinese)]. Zhonghua Bing Li Xue Za Zhi. 2019; 48(3):186-91. [PMID]

[21] Saadeh FS, Mahfouz R, Assi HI. EGFR as a clinical marker in glioblastomas and other gliomas. The International Journal of Biological Markers. 2018; 33(1):22-32. [DOI:10.5301/ ijbm.5000301] [PMID]

[22] Fisher JL, Schwartzbaum JA, Wrensch M, Wiemels JL. Epidemiology of brain tumors. Neurologic Clinics. 2007; 25(4):867-90. [DOI:10.1016/j.ncl.2007.07.002] [PMID]

[23] Dohrmann GJ, Farwell JR, Flannery JT. Glioblastoma multiforme in children. Journal of Neurosurgery. 1976; 44(4):4428. [DOI:10.3171/jns.1976.44.4.0442] [PMID]

[24] Shuangshoti S, Panyathanya R. Glioblastoma multiforme: Study of 174 cases. Journal of the Medical Association of Thailand. 1983; 66(3):150-65. [PMID]

[25] Monzón MJ, Pascual-Piazuelo MC, López-López A, Calatayud V, Eiras J. [Incidence of glioblastoma multiforme in Aragon and La Rioja. An epidemiological survey (Spanish)]. Revista de Neurologia. 1996; 24(125):73-6. [PMID]

[26] Tomlinson FH, Kurtin PJ, Suman VJ, Scheithauer BW, O'Fallon JR, Kelly PJ, et al. Primary intracerebral malignant lymphoma: A clinicopathological study of 89 patients. Journal of Neurosurgery. 1995; 82(4):558-66. [DOI:10.3171/ jns.1995.82.4.0558] [PMID] 